\title{
Electrospun gelatin/PCL and collagen/PLCL scaffolds for vascular tissue engineering
}

This article was published in the following Dove Press journal:

International Journal of Nanomedicine

13 May 2014

Number of times this article has been viewed

\author{
Wei $\mathrm{Fu}^{1,2, *}$ \\ Zhenling Liu',* \\ Bei Feng ${ }^{1,2}$ \\ Renjie $\mathrm{Hu}^{\prime}$ \\ Xiaomin $\mathrm{He}^{1}$ \\ Hao Wang' \\ Meng Yin' \\ Huimin Huang' \\ Haibo Zhang' \\ Wei Wang' \\ 'Department of Pediatric \\ Cardiothoracic Surgery, ${ }^{2}$ Institute \\ of Pediatric Translational Medicine, \\ Shanghai Children's Medical Center, \\ Shanghai Jiao Tong University School \\ of Medicine, Shanghai, People's \\ Republic of China \\ *These authors contributed equally \\ to this work
}

\begin{abstract}
Electrospun hybrid nanofibers prepared using combinations of natural and synthetic polymers have been widely investigated in cardiovascular tissue engineering. In this study, electrospun gelatin/polycaprolactone (PCL) and collagen/poly(l-lactic acid-co- $\varepsilon$-caprolactone) (PLCL) scaffolds were successfully produced. Scanning electron micrographs showed that fibers of both membranes were smooth and homogeneous. Water contact angle measurements further demonstrated that both scaffolds were hydrophilic. To determine cell attachment and migration on the scaffolds, both hybrid scaffolds were seeded with human umbilical arterial smooth muscle cells. Scanning electron micrographs and MTT assays showed that the cells grew and proliferated well on both hybrid scaffolds. Gross observation of the transplanted scaffolds revealed that the engineered collagen/PLCL scaffolds were smoother and brighter than the gelatin/PCL scaffolds. Hematoxylin and eosin staining showed that the engineered blood vessels constructed by collagen/PLCL electrospun membranes formed relatively homogenous vessel-like tissues. Interestingly, Young's modulus for the engineered collagen/PLCL scaffolds was greater than for the gelatin/PCL scaffolds. Together, these results indicate that nanofibrous collagen/PLCL membranes with favorable mechanical and biological properties may be a desirable scaffold for vascular tissue engineering.
\end{abstract}

Keywords: electrospinning, gelatin, collagen, polycaprolactone, poly(1-lactic acid-co- $\varepsilon$ caprolactone)

\section{Introduction}

Recently, synthetic vascular grafts made from expanded polytetrafluoroethylene or polyethylene terephthalate $\left(\operatorname{Dacron}^{\circledR}\right)$ have been successfully used to replace large diameter blood vessel ( $\geq 6 \mathrm{~mm}$ ) implants in clinical studies. ${ }^{1}$ However, taking the risks of thrombosis, calcification, and restenosis into consideration, they are unsuitable replacements for small diameter blood vessels $(<6 \mathrm{~mm}){ }^{2}$ In addition, the synthetic materials used in clinical replacement procedures have been found to lack growth potential, which is critical for children, and often require a second surgery to replace the implants. ${ }^{3}$ Autologous or allogeneic transplantation is widely used to reconstruct small diameter blood vessels at present. ${ }^{4,5}$ Unfortunately, due to lack of donors and potential immunogenicity issues, this strategy often cannot meet the requirements for clinical studies. Therefore, use of biodegradable materials for vascular reconstructions could provide significant benefits over other known techniques. Acceptable mechanical properties are vital considerations, since the implant will undergo continuous blood flow and vessel movement in vivo. ${ }^{6-8}$ Furthermore, an appropriate biodegradation 
profile will be required for native tissue growth and biocompatibility for the effective regeneration of functional tissue.

Among the currently known fabrication techniques, electrospinning has been shown to be an effective approach to produce nanofibrous scaffolds. It provides a biomimetic cellular environment that resembles the extracellular matrix of native tissues. Data indicate that electrospinning facilitates cell growth, allows for efficient exchange of nutrients and metabolic wastes between scaffolds and their environment, and provides a large surface area for the delivery of biochemical signals to the seeded cells. ${ }^{9-11}$ To date, a variety of natural and synthetic electrospun polymers have been used extensively to prepare vascular scaffolds. ${ }^{12-14}$

Natural polymers have often been used to prepare vascular scaffolds based on their biocompatibility profiles. For example, collagen and elastin (the primary structural components of the extracellular matrix in vascular tissues) have been electrospun into fibrous scaffolds for vascular grafting applications. ${ }^{15,16}$ These electrospun protein scaffolds have been shown to successfully promote cell adhesion, migration, and proliferation. However, scaffolds prepared from natural components alone fail to achieve the desired mechanical characteristics, being unable to retain their structural integrity and swelling in aqueous environments. ${ }^{12,17}$ On the other hand, synthetic electrospun scaffolds containing materials such as polycaprolactone (PCL), poly(L-lactide-co- $\varepsilon$ caprolactone) (PLCL), and poly(L-lactide-co-glycolide) have been shown to have good mechanical strength and tunable biodegradability. ${ }^{13,18,19}$

Interestingly, more promising results have been achieved by combining natural and synthetic polymers. Previous reports indicate that an electrospun scaffold containing both PCL and collagen had excellent tensile strength $(4.0 \pm 0.4 \mathrm{MPa})$ and burst pressure $(4,912 \pm 155 \mathrm{mmHg})$, comparable with native vessels. ${ }^{7,20}$

In the current study, we generated electrospun hybrid membranes containing synthetic and natural materials, ie, gelatin/PCL and collagen/PLCL. These membranes were then characterized by scanning electron microscopy (SEM), fiber diameter distribution, water contact angle measurements, and mechanical analysis. We further examined the biocompatibility of these scaffolds using human umbilical arterial smooth muscle cells (HUSMCs) by evaluating their morphology, viability, and proliferation. Finally, we constructed a vascular scaffold with an inner diameter of $4 \mathrm{~mm}$ and implanted it subcutaneously into nude mice for a 6-week in vivo analysis.

\section{Materials and methods \\ Fabrication of gelatin/PCL and collagen/PLCL scaffolds}

Composite fibers of gelatin/PCL (50:50) were fabricated as described previously. ${ }^{21}$ Briefly, gelatin type A (300 Bloom from porcine skin in powder form, SigmaAldrich, St Louis, MO, USA) and PCL (molecular weight 80,000 , Sigma-Aldrich) were dissolved separately in 2,2,2-trifluoroethanol (TFE, purity $\geq 99.0 \%$, SigmaAldrich) at a concentration of $10 \%(\mathrm{w} / \mathrm{v})$ with sufficient stirring at room temperature for 24 hours. Before electrospinning, the two solutions were mixed in 50:50 volume ratios. To prepare the transparent gelatin/PCL/TFE solution, a small amount of acetic acid $(0.2 \%$ with respect to TFE) was dropped into the above prepared solutions. Composite fibers of collagen/PLCL (50:50) were fabricated as described previously. ${ }^{19}$ Briefly, type I collagen (Sichuan Mingrang Biotechnology Co, Sichuan, People's Republic of China) and PLCL (Nara Medical University, Kashihara, Japan), which has a composition of $50 \mathrm{wt} \%$ L-lactide and $50 \mathrm{wt} \% \varepsilon$-caprolactone monomers, were dissolved separately in hexafluoroisopropanol (purity $\geq 99.0 \%$, SigmaAldrich) at a concentration of $8 \mathrm{wt} \%$ and stirred vigorously at room temperature for 24 hours. Before electrospinning, the two solutions were mixed in 50:50 volume ratios. The two groups of solution were fed into a $10 \mathrm{~mL}$ plastic syringe and electrospun using the parameters defined in Table 1. A KDS100 syringe pump (KD Scientific, Holliston, MA, USA) and a high voltage power supply (TXR1020N30-30, Teslaman, Dalian, People's Republic of China) were used to control the solution dispensing rate and applied voltage, respectively. The prepared collagen/PLCL fibrous membranes were dried in a vacuum oven for 1 week at room temperature to remove residual solvent before subsequent use.

Table I Parameters used for electrospinning fibrous membrane

\begin{tabular}{lllllllr}
\hline Sample & Solvent & Concentration & $\begin{array}{l}\text { Applied } \\
\text { voltage } \mathbf{( k V})\end{array}$ & $\begin{array}{l}\text { Feed rate } \\
(\mathbf{m L} / \mathbf{h})\end{array}$ & $\begin{array}{l}\text { Collecting } \\
\text { distance } \mathbf{( c m})\end{array}$ & Temperature Humidity \\
\hline GT/PCL (50:50) & TFE & $10 \mathrm{w} / \mathrm{v} \%$ & 10 & 2 & 13 & $20^{\circ} \mathrm{C}-25^{\circ} \mathrm{C}$ & $40 \%-60 \%$ \\
Collagen/PLCL (50:50) & HFIP & $8 \mathrm{w} / \mathrm{v} \%$ & 5 & 1 & 13 & $20^{\circ} \mathrm{C}-25^{\circ} \mathrm{C}$ & $40 \%-60 \%$ \\
\hline
\end{tabular}

Abbreviations: PCL, polycaprolactone; PLCL, poly(l-lactic acid-co-e-caprolactone); TFE, 2,2,2-trifluoroethanol; GT, gelatin; HFIP, hexafluoroisopropanol. 


\section{Characterization of scaffolds}

The morphology of the electrospun gelatin/PCL and collagen/ PLCL fibers was observed by SEM (JSM-5600LV, JEOL Ltd, Tokyo, Japan) operated at an acceleration voltage of 8-10 kV. Before observation, the scaffolds were coated with gold for 50 seconds to increase conductivity. Fiber diameters were measured using ImageJ 1.40G software (National Institutes of Health, Bethesda, MD, USA). At least 100 nanofibers from each sample were analyzed in different SEM images.

The hydrophilicity of the electrospun gelatin/PCL and collagen/PLCL fibrous membranes was determined using water contact angle measurements in order to give an indication of the wetting ability of the scaffold. The contact angle was measured using a video contact angle instrument (Attension Theta, Espoo, Finland). Droplets of $0.5 \mu \mathrm{L}$ were dispensed onto the scaffold and the contact angle was determined automatically.

The mechanical properties of the electrospun fibrous membranes were determined using a tabletop uniaxial material testing machine (Instron-3343, Instron, Norwood, USA) equipped with a $50 \mathrm{~N}$ load cell. Rectangular-shaped specimens $(30 \mathrm{~mm} \times 10 \mathrm{~mm} \times 0.10-0.20 \mathrm{~mm})$ were stretched at a constant cross-head speed of $10 \mathrm{~mm}$ per minute. Five samples in each group were tested. For each specimen, the greatest slope in the linear region of the stress-strain curve corresponding to strain of $0 \%-20 \%$ was used to calculate the Young's modulus.

\section{Cell isolation and culture}

Cell isolation, culture, and identification were initiated after the study had been approved by the ethics committee at Shanghai Children's Medical Center and informed consent had been obtained for all tissues subsequently used. HUSMCs were isolated from a healthy newborn umbilical cord and identified according to methods described elsewhere. ${ }^{22}$ The cells were then cultured in Dulbecco's Modified Eagle's Medium (HyClone, Logan, UT, USA) with 10\% fetal bovine serum (Hyclone) and 1\% penicillin-streptomycin (Gibco, Grand Island, NY, USA). The medium was changed every 3 days.

\section{Immunofluorescence staining}

Immunofluorescence staining was carried out using standard procedures as described previously. ${ }^{23}$ Rabbit polyclonal to alpha smooth muscle actin and rabbit polyclonal to smooth muscle myosin heavy chain 2 were purchased from Abcam (ab5694, ab53219; Cambridge, UK). Anti-rabbit IgG (H+L) was purchased from Invitrogen (HH-A21207; Carlsbad, CA, USA). We did not add the primary antibody and only added the second antibody in the control group. Cells were observed using an IX50 fluorescence microscope (Olympus, Tokyo, Japan) after staining.

\section{SEM observation}

Cells were seeded onto the gelatin/PCL and collagen/ PLCL fiber scaffolds in 24-well plates at a seeding density of $2 \times 10^{4}$ cells/well. One day after seeding, cell morphology was visualized using SEM. Specimens were fixed in $0.25 \%$ glutaraldehyde for 30 minutes, rinsed three times in phosphate-buffered saline, then dehydrated in graded concentrations of ethanol at 30\%, 50\%, 70\%, 80\%, 90\%, and $100 \%$ for 10 minutes each. Finally, they were air-dried in a fume hood overnight. After drying, the specimens were coated with gold sputter and observed by SEM (6380LV, JEOL Ltd) at an accelerating voltage of 8-10 kV.

\section{Cell proliferation assay}

To study cell viability and proliferation on the different substrates, HUSMCs were also seeded onto the scaffolds in 24-well plates at a density of $2 \times 10^{4}$ cells/well and quantified using a Cell Counting Kit-8 (CCK-8, Dojindo Molecular Technologies Inc, Rockville, MD, USA). At desired time points, the cell-seeded scaffolds were incubated in $10 \%$ CCK -8 at $37^{\circ} \mathrm{C}$ for 2 hours in $5 \% \mathrm{CO}_{2}$. The absorbance of each well was measured at $450 \mathrm{~nm}$ using a microplate reader (Multiskan MK3, Thermo Electron Corporation, MA, USA). At least six samples were measured at each time point.

\section{Engineering of cell-scaffold constructs}

The membranes were cut into rectangles $(2 \mathrm{~cm} \times 7.5 \mathrm{~cm})$ with an average thickness of $20 \mu \mathrm{m}$, lyophilized in a Virtis Benchtop 6.6 vacuum freeze-dryer (SP Industries, Gardiner, NY, USA) for 24 hours, sterilized for 30 minutes under ultraviolet radiation, and then placed in the bottom of $10 \mathrm{~cm}$ culture dishes seeded with $100 \mu \mathrm{L}$ of cell suspension at $1 \times 10^{8}$ cells $/ \mathrm{mL}$. To ensure proper adhesion between cells and the materials, the constructs were kept in the incubator without culture medium (Dulbecco's Modified Eagle's Medium with $10 \%$ of fetal calf serum) for 30 minutes. A sterilized tube (length $3 \mathrm{~cm}$, diameter $4 \mathrm{~mm}$ ) was placed on the material and the cell-membrane complexes were rolled around the tube. Culture medium $(10 \mathrm{~mL})$ was added to the dish along the wall and the cell-scaffold complexes were placed in the incubator for 1 week. The medium was changed every 3 days.

\section{Gross observation}

The cell-scaffold constructs were then implanted into nude mice (obtained from the Shanghai Laboratory Animal 
Center, Chinese Academy of Sciences, People's Republic of China) which were anesthetized using $2.5 \%$ chloral hydrate. The skin of the dorsum was cut at approximately $2 \mathrm{~cm}$ by dissecting one side of the subcutaneous fascia, and the cellscaffold constructs were implanted subcutaneously. Six weeks after implantation, the mice were euthanized and the cellscaffold constructs were dissected for further investigation. Each construct was observed grossly for shape, color, and size, and palpated with forceps to assess mechanical strength.

\section{Histological findings}

Following gross examination, samples were fixed in $4 \%$ paraformaldehyde, embedded in paraffin, and sectioned into $5 \mu \mathrm{m}$ sections. The sections were prepared for histological analysis by staining with hematoxylin and eosin and Masson's trichrome. Newborn pig aortic blood vessels were used for normalization.

\section{Biomechanical analysis}

The mechanical properties of the electrospun fibrous scaffolds in the wet state were measured using a biomechanical testing machine (Instron-3343; Norwood) as previously described in the literature. ${ }^{27}$ Samples were tailored to rectangular-shaped specimens $(30 \mathrm{~mm} \times 10 \mathrm{~mm} \times 1-1.5 \mathrm{~mm})$, and stretched at a constant cross-head speed of $5 \mathrm{~mm}$ per minute with a $50 \mathrm{~N}$ load cell. For testing samples in the wet state, the fibrous membranes were sterilized and soaked in phosphate-buffered saline for 12 hours. Five samples in each group were tested. The mechanical properties of the cell-scaffold constructs were determined using the same method. Samples were tailored to rectangular-shaped specimens $(10 \mathrm{~mm} \times 5 \mathrm{~mm}$ $\times 1-1.5 \mathrm{~mm}$ ) and immersed in phosphate-buffered saline for 12 hours at room temperature before the test. For each specimen, the greatest slope in the linear region of the stress-strain curve corresponding to strain between $0 \%$ and $20 \%$ was used to calculate the tensile modulus.

\section{Statistical analysis}

All data presented are expressed as the mean \pm standard deviation. The statistical analysis was carried out using single-factor analysis of variance. A value of $P<0.05$ was considered to be statistically significant.

\section{Results}

\section{Morphology of the nanofibrous scaffold}

Composite gelatin/PCL scaffolds with a mass ratio of 50:50 were prepared using the electrospun method described above. As can be seen in Figure 1A-C, the nanofibers appear uniform and smooth with a mean diameter of $386.9 \pm 102.5 \mathrm{~nm}$ (ImageJ). The electrospun collagen/PLCL fibrous membranes with a mass ratio of 50:50 also appeared smooth and uniform, as can be seen in Figure 1D-F. The fiber diameter was estimated to be smaller than that of the gelatin/PCL at 301.8 \pm 97.3 $\mathrm{nm}$. Together, these results indicate that the blended gelatin/ PCL and collagen/PLCL fibers were successfully engineered without beads.

\section{Water contact angle measurements}

Surface wettability is an important property of biomaterials and can affect the attachment, proliferation, migration, and viability of cells. ${ }^{24}$ The water contact angle can be used to evaluate wettability. Most synthetic polymers, including PCL
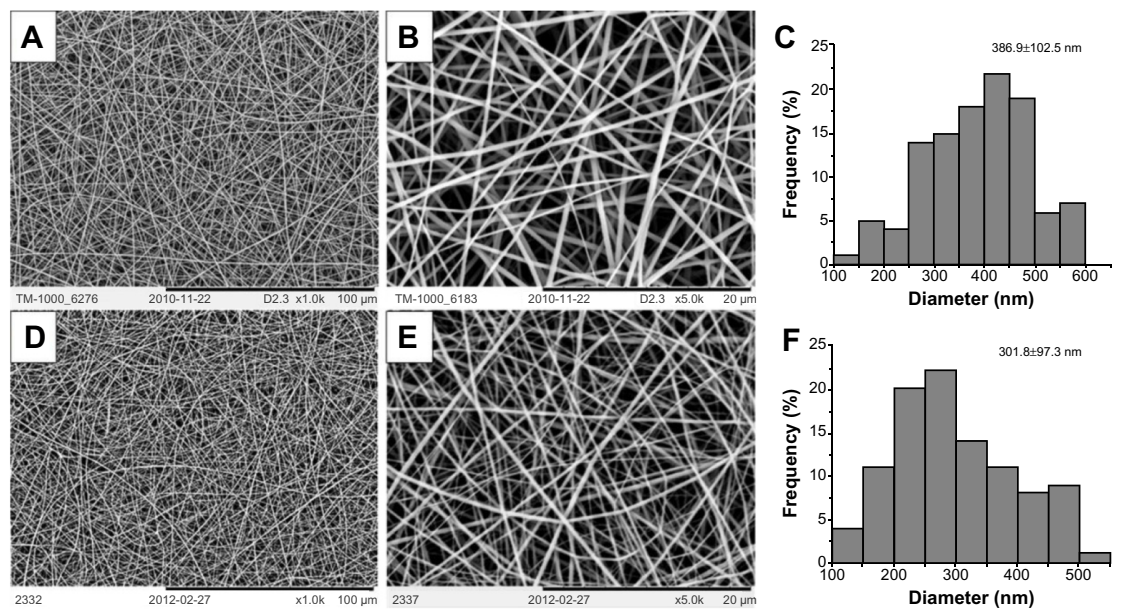

Figure I SEM of electrospun nanofiber membranes.

Notes: Gelatin/PCL (A 1000×, B 5000×), collagen/PLCL (D 1000×, E 5000×), and diameter distributions of gelatin/PCL (C), collagen/PLCL (F) membranes. Abbreviations: PCL, polycaprolactone; PLCL, poly(l-lactic acid-co- $\varepsilon$-caprolactone); SEM, scanning electron microscope. 
and PLCL, are hydrophobic, whereas collagen and gelatin (a product of partially hydrolyzed collagen) are hydrophilic. In order to form hydrophilic nanofibers, an appealing approach has been to process naturally derived polymers into synthetic polymers. After the gelatin or collagen was combined with PCL or PLCL to form hybrid nanofibers, we observed that the water drop was immediately absorbed into the fibrous networks, resulting in a zero contact angle at 10 seconds, which indicated that the gelatin/PCL and collagen/PLCL electrospun membranes were in fact hydrophilic and could be used successfully for cell seeding (Figure 2).

\section{Identification and characterization of HUSMCs}

Immunofluorescence staining of alpha smooth muscle actin and smooth muscle myosin heavy chain 2 was used to identify the HUSMCs. As can be seen in Figure 3, both proteins were expressed in HUSMCs at cell passage 3. Cells from passages 3-5 were used for the following experiments.

\section{Proliferation and morphology of HUSMCs on the scaffolds}

Cell adhesion and proliferation are critical parameters when evaluating whether a scaffold is biocompatible and suitable for tissue regeneration. SEM was used to observe cell morphology and the contacts between the cells and scaffolds. As shown in Figure 4A and B, HUSMCs were strongly adhered and well spread on both scaffolds after 1 day of cell seeding. Cells exhibited a bipolar spindle shape, indicating a contractile phenotype. A CCK-8 test was carried out to observe the growth and proliferation of HUSMCs on different scaffolds and the tissue culture plate. HUSMCs proliferated well and the number of HUSMCs increased with culture time on all the three substrates, indicating that the scaffolds were nontoxic. Significantly more cells were observed on the tissue culture plate than on the gelatin/PCL or collagen/PLCL scaffolds. However, there was no statistically significant difference in cell numbers between the gelatin/PCL and collagen/PLCL scaffolds (Figure 4C). On the first day following seeding, significantly more cells were found on the tissue culture plate than on the collagen/PLCL scaffolds, and the gelatin/ PCL substrate had significantly less adherent cells than the other two substrates (Figure 4D, $P<0.05$ ). Together, these results indicate that the gelatin/PCL and collagen/PLCL scaffolds had good biocompatibility with HUSMCs, further suggesting their promising potential for regeneration of cardiovascular tissue.

\section{Gross observation}

The electrospun gelatin/PCL and collagen/PLCL membranes were first tailored into rectangles $(2 \mathrm{~cm} \times 7.5 \mathrm{~cm})$ with an average thickness of $20 \mu \mathrm{m}$ (Figure 5A) and then sterilized and placed in the bottom of $10 \mathrm{~cm}$ culture dishes seeded with $100 \mu \mathrm{L}$ of cell suspension at $1 \times 10^{8}$ cells $/ \mathrm{mL}$ (Figure $5 \mathrm{~B}$ ). The complexes were placed in the incubator for 30 minutes without culture medium and then rolled up with a catheter (Figure 5C). The cell-scaffold construct maintained its original shape after 1 week of culture in vitro. As expected, few adherent cells were observed on the bottom of the culture dish after 24 hours (data not shown), indicating that the majority of cells were contained within the constructs. After 1 week of in vitro culture, the cell-scaffold constructs were implanted subcutaneously in nude mice for another 6 weeks (Figure 5E). As shown in Figure 5E-L, the constructs were very hard to dissect from the surrounding soft connective tissue and covered with many small blood vessels. However, there was no evidence of superficial inflammatory reactions, and all of the constructs maintained their original shape and tubular appearance. The collagen/PLCL membranes (Figure 5F and $\mathrm{J}-\mathrm{L}$ ) appeared smooth and glossy with good

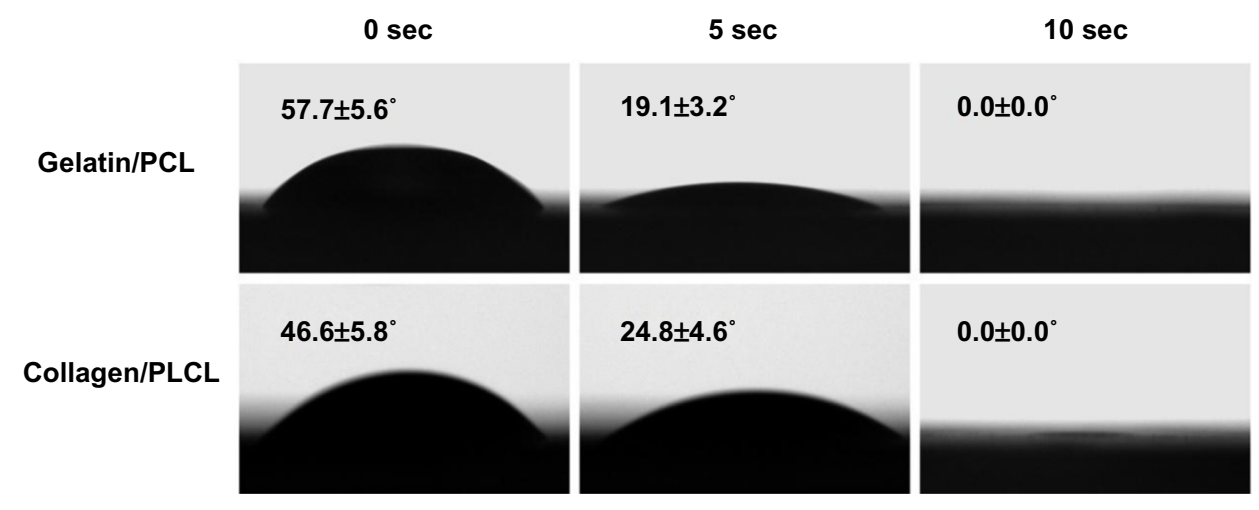

Figure 2 Water contact angles of gelatin/PCL and collagen/PLCL electrospun fibrous membranes. Abbreviations: PCL, polycaprolactone; PLCL, poly(l-lactic acid-co- $\varepsilon$-caprolactone). 

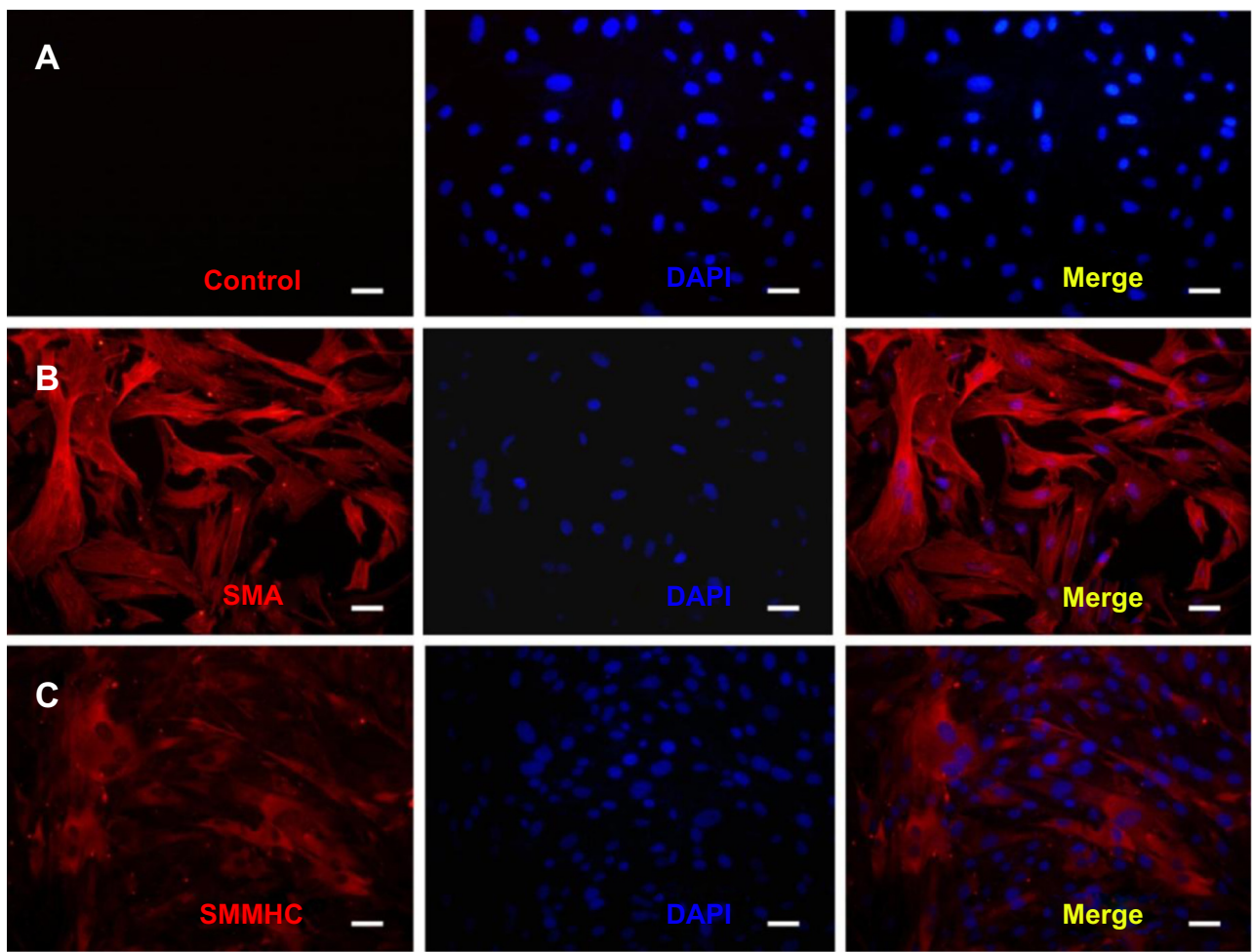

Figure 3 Immunofluorescence staining of human umbilical arterial smooth muscle cells (HUSMCs).

Notes: (A) Control; (B) immunofluorescence staining of HUSMCs for alpha smooth muscle actin (SMA); (C) immunofluorescence staining of HUSMCs for smooth muscle myosin heavy chain 2 (SMMHC).

Abbreviation: DAPI, 4',6-diamidino-2-phenylindole.
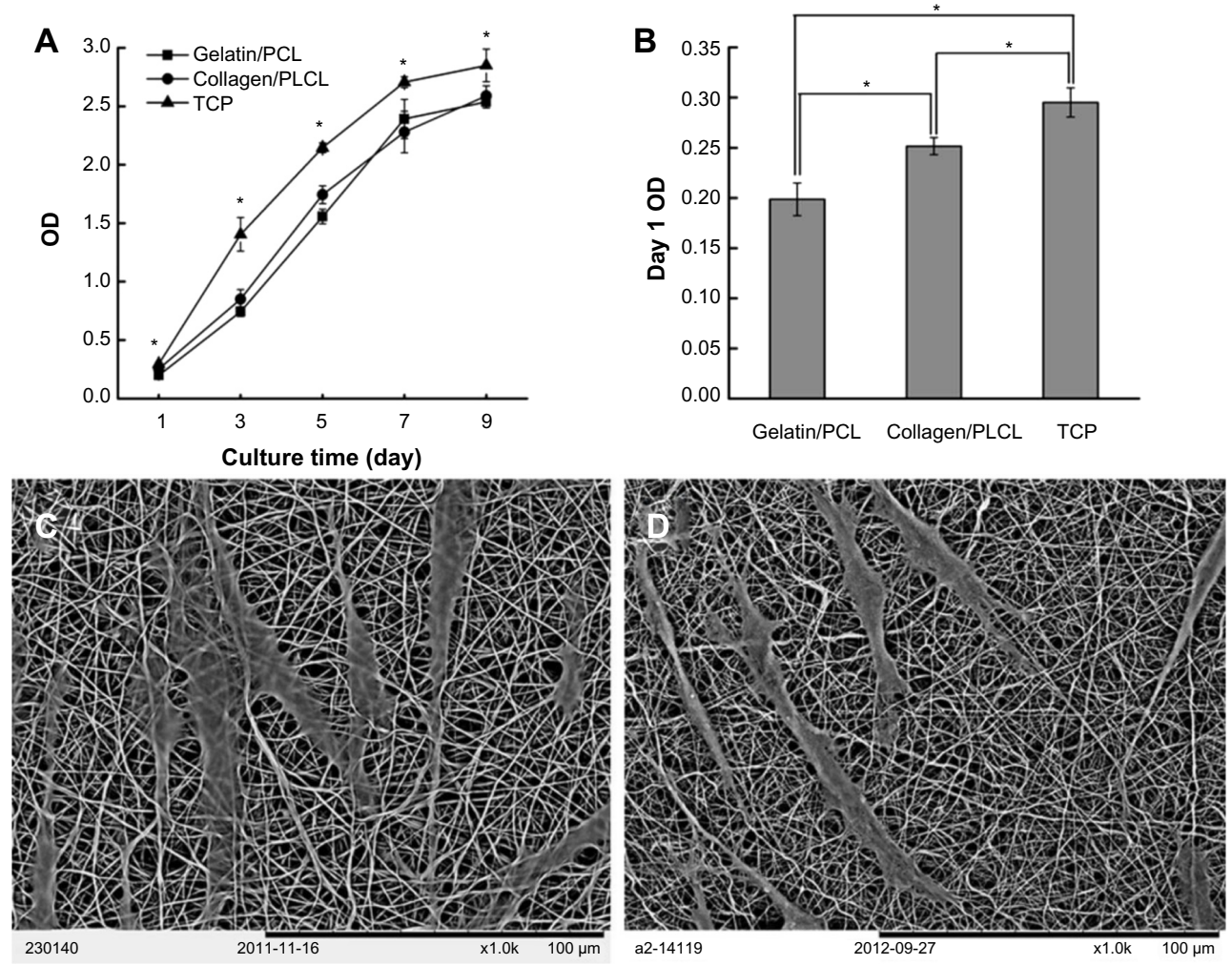

Figure 4 Adherence and proliferation of HUSMCs on various materials.

Notes: SEM images of HUSMCs grown on gelatin/PCL (A) and collagen/PLCL (B) electrospun fibrous membranes for I day. Cell proliferation (C) and adhesion rate (D) on various materials measured by a CCK-8 kit.

Abbreviations: PCL, polycaprolactone; PLCL, poly(l-lactic acid-co- $\varepsilon$-caprolactone); SEM, scanning electron microscope; HUSMC, human umbilical arterial smooth muscle cell; CCK-8, Cell Counting Kit-8; TCP, tissue culture plate; OD, optical density. 

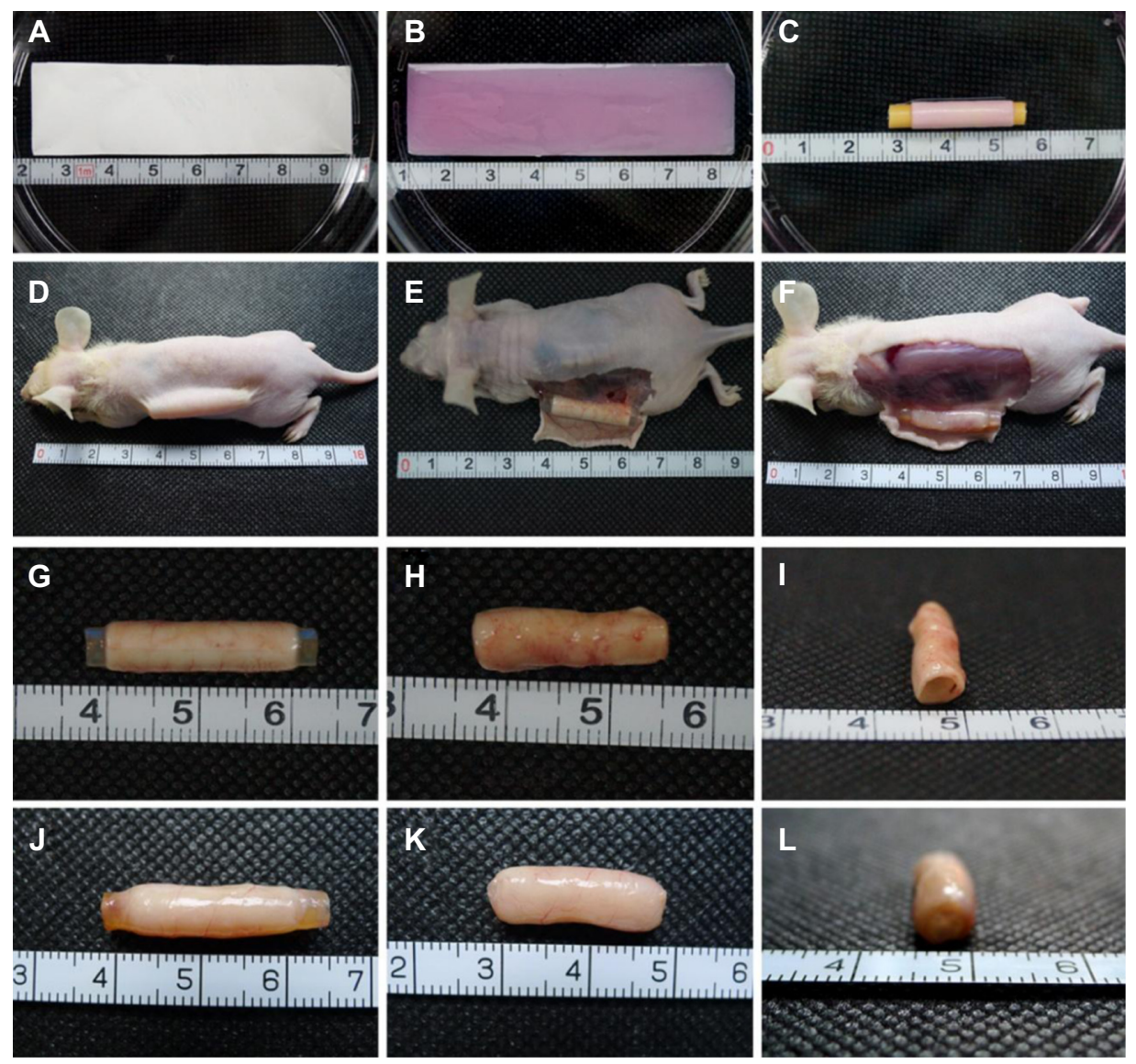

Figure 5 Gross view of cell scaffold constructs.

Notes: (A) Tailored electrospun fibrous membranes. (B) Gross view of cell-scaffold constructs. (C) Cell-scaffold constructs rolled up with a catheter. (D) Subcutaneous implantation in nude mice. (E and $\mathbf{G}-\mathbf{I}$ ) Cell scaffold constructs after 6 weeks in vivo incubation with gelatin/PCL. (F and J-L) Cell scaffold constructs after 6 weeks in vivo incubation with collagen/PLCL.

Abbreviations: $\mathrm{PCL}$, polycaprolactone; PLCL, poly(l-lactic acid-co- $\varepsilon$-caprolactone).

elasticity, while the gelatin/PCL membranes (Figure 5E and G-I) were relatively soft and easily collapsed when the inner catheter was removed, indicating inferior tissue formation.

\section{Histological analysis}

The results of histological imaging (Figure 6) were consistent with the gross morphological analysis. The engineered blood vessels constructed by collagen/PLCL electrospun membranes formed relatively homogenous vessel-like tissues with bands of collagen fibers (Figure 6B, E, H, K, and N). A lot of microvessels formed in the engineered blood vessels, and red blood cells could be seen in these microvessels (Figure $6 \mathrm{H}$ and $\mathrm{N}$ ). Hematoxylin and eosin staining showed that the cells had a spindle-shaped morphology, formed a linear distribution along the circumference of the engineered blood vessels, and almost no nondegraded scaffolds were observed. However, the samples constructed by gelatin/PCL electrospun membranes (Figure 6A, D, G, J, and K) showed an obviously heterogeneous structure with relatively fewer collagen fibers, and only a small amount of microvascular structures have formed along the walls of the blood vessels (Figure 6G and $\mathrm{M}$ ). In addition, there were abundant nondegraded scaffolds observed and the remaining scaffolds showed an irregular zonal distribution, indicating that the gelatin/PCL electrospun membranes was unfavorable for vessel-like tissue formation and matrix production.

\section{Mechanical properties of the scaffolds}

Desirable mechanical properties for the scaffolds are especially important because of the forces they would endure in vivo after implantation. Young's modulus was determined to be $1.49 \pm 0.06 \mathrm{MPa}$ and $1.77 \pm 0.09 \mathrm{MPa}$ for the gelatin/PCL and collagen/PLCL membranes, respectively (Figure 7). These values are notably higher than the $1 \mathrm{MPa}$ reported for the porcine coronary artery. ${ }^{7} \mathrm{Six}$ weeks following implantation, the value for the gelatin/PCL membranes decreased to $0.75 \pm 0.15 \mathrm{MPa}$, suggesting rapid degradation of the gelatin and lack of tissue formation (Figure 7). Young's modulus for the collagen/PLCL scaffolds, however, increased 6 weeks post implantation to 


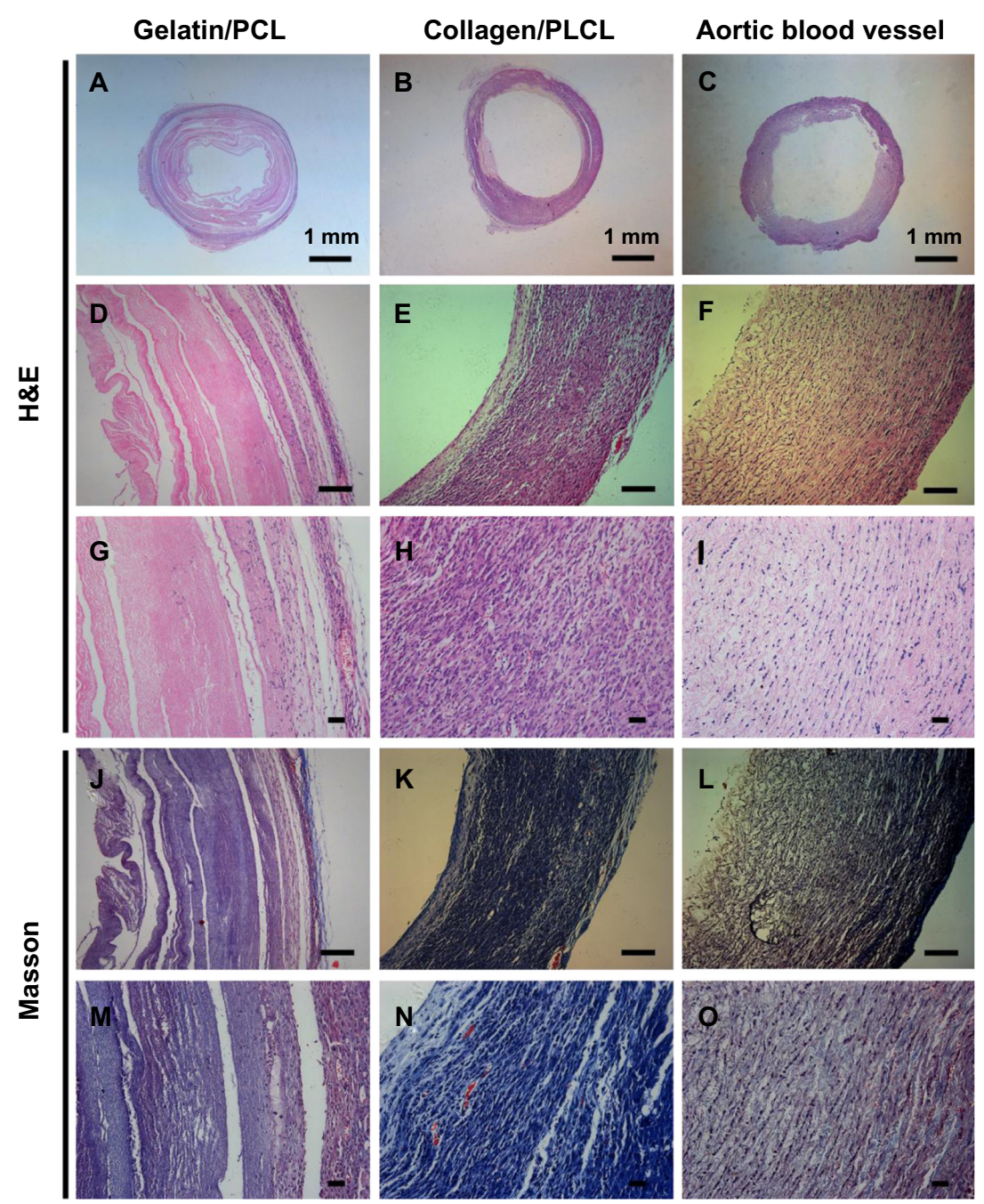

Figure 6 Histological images of cell scaffold constructs with H\&E and Masson's trichrome staining at 6 weeks in vivo.

Notes: Panels A, D, G, J and $\mathbf{M}$ appear more heterogeneous with a large number of non-degraded scaffolds and less collagen fibers. Panels B, E, H, K and $\mathbf{N}$ show relatively homogenous vessel-like tissue structures with bands of collagen fibers formed. A newborn pig aortic blood vessel was used as a positive control (C, F, I, $\mathbf{L}$ and $\mathbf{O}$ ). Scale bars: $100 \mu \mathrm{m}$.

Abbreviations: H\&E, hematoxylin and eosin; PCL, polycaprolactone; PLCL, poly(l-lactic acid-co- $\varepsilon$-caprolactone).

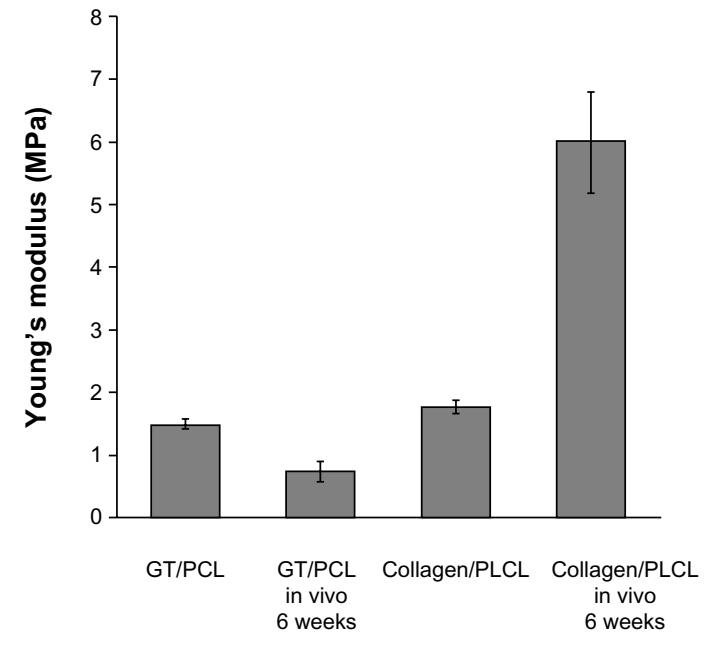

Figure 7 Young's modulus of electrospun nanofiber membranes and cell scaffold constructs in vitro and in vivo.

Note: Bars represent mean \pm standard deviation $(n=6)$.

Abbreviations: PCL, polycaprolactone; PLCL, poly(l-lactic acid-co- $\varepsilon$-caprolactone); GT, gelatin.
$5.99 \pm 0.80 \mathrm{MPa}$, likely because of enhanced vessel-like tissue formation (Figure 7).

\section{Discussion}

Scaffolds play an important role in directing three-dimensional tissue regeneration. ${ }^{25,26}$ Electrospinning using natural proteins or synthetic polymers is an effective method for fabricating scaffolds that mimic the nanostructures of the natural extracellular matrix. Electrospun gelatin/PCL and collagen/ PLCL membranes have been widely used in various tissue engineering studies because of their hydrophilicity, excellent mechanical properties, and biocompatibility. ${ }^{27-30}$ However, it is still unknown which material is most appropriate for vascular tissue regeneration.

In this study, gelatin/PCL and collagen/PLCL hybrid scaffolds were successfully prepared using electrospinning techniques. Gelatin and collagen are natural hydrophilic 
biopolymers that have been shown to enhance the wettability of membranes. PCL and PLCL are synthetic polymers that provide good mechanical strength. Both of the hybrid scaffold materials used in this study were shown to be hydrophilic and to possess excellent mechanical properties, likely due to the combined merits of natural and synthetic polymers. Interestingly, no obvious differences were observed between the groups with regard to cell attachment or proliferation. SEM and CCK-8 showed good biocompatibility in both groups. The electropsun nanofibers generated in this study successfully mimicked the properties of extracellular matrix tissues previously shown to facilitate cell adhesion and proliferation. However, in vivo vascular tissue regeneration experiments showed inconsistent results. For example, despite sufficient biocompatibility, the regenerated vascular tissue showed differing appearances and structures in the two groups. At 6 weeks post implantation in vivo, the collagen/ PLCL electrospun scaffolds appeared smooth and glossy with good elasticity, while the gelatin/PCL electrospun scaffolds were relatively soft and easily collapsible based on gross histology. The scaffolds constructed by collagen/ PLCL electrospun membranes formed relatively homogenous vessel-like tissues with bands of collagen fibers. The scaffolds constructed from gelatin/PCL membranes appeared more heterogeneous with a large number of nondegraded scaffolds and relatively less collagen fiber formation. These data suggest that collagen/PLCL electrospun membranes may be preferable for vascular tissue regeneration.

Material degradation speed is an important factor to consider in tissue engineering. ${ }^{31-33}$ Degradation of the scaffold should not be so rapid that cells do not have enough time to proliferate and secrete the new matrix. However, if scaffold degradation is much slower than tissue regeneration, the nondegraded scaffold will hamper deposition of extracellular matrix and thus influence the homogeneity and biological function of the regenerated tissue. ${ }^{34}$ With regard to selection of materials, pure gelatin or collagen has been shown to be unsuitable for vascular tissue regeneration based on factors related to structural integrity. ${ }^{12,17}$ Previous reports indicate that degradation of PCL is slower than that of PLCL. ${ }^{35,36}$ Our results showed abundant nondegraded scaffolds in the gelatin/PCL group 6 weeks post implantation in addition to the presence of heterogeneous structures and relatively less collagen fiber formation. These data suggest that the delayed degradation of the scaffold significantly hampered the regeneration of vascular tissue and that the PCL scaffold might be unfavorable for formation of vascular tissue.

Post-implantation nutrition was also speculated to be a critical determinant of the success of vascular tissue formation. ${ }^{37}$ As described in the "Methods" section, a catheter was used to support the shape of the scaffold. Following implantation, the construct received most of its nutrients from the surface-connected skin and not from the inner surface of the tube. The abundant continuous nondegraded scaffold in the gelatin/PCL group not only hindered regeneration of vascular tissue (as mentioned above) but also further hampered the exchange of nutrients and metabolic waste between the scaffold and its environment. These factors may have played a role in the lack of formation of vascular tissue in the gelatin/PCL scaffolds.

\section{Conclusion}

In this study, gelatin/PCL and collagen/PLCL hybrid scaffolds were successfully prepared using electrospun techniques. The scaffolds showed desirable mechanical properties, wettability, and the ability to promote cell adhesion and proliferation. Six weeks following subcutaneous in vivo implantation into nude mice, the scaffolds constructed using collagen/PLCL membranes appeared to be relatively homogenous with vessel-like tissues formed from bands of collagen fibers. The scaffolds constructed from gelatin/PCL membranes appeared more heterogeneous with abundant nondegraded scaffolds and relatively less collagen fiber formation. The current study suggests that electrospun collagen/PLCL membranes may be preferable for regeneration of vascular tissue.

\section{Acknowledgments}

This study was supported by the National Natural Science Fund of China (31200735, 81271726,80170151), the Shanghai Science and Technology Project (13ZR1426400, 134119a0400), the Shanghai Natural Science Fund for Youth Scholars (12ZR1446500), the Science and Technology Development Fund of Shanghai Pudong (PKJ2011-Y320, PKJ-Y30), the Biomedical Engineering Fund of Shanghai Jiao Tong University (YG2012MS36, YG2012MS35, YG2011ZD03), the College Young Teachers' Training and Funding Project of Shanghai (ZZjdyx12117, ZZjdyx12124), and the College Young Teachers' Training and Funding Project of Shanghai Jiao Tong University School of Medicine.

\section{Disclosure}

The authors report no conflicts of interest in this work.

\section{References}

1. Hoenig MR, Campbell GR, Rolfe BE, Campbell JH. Tissue-engineered blood vessels: alternative to autologous grafts? Arterioscler Thromb Vasc Biol. 2005;25(6):1128-1134.

2. He W, Ma Z, Teo WE, et al. Tubular nanofiber scaffolds for tissue engineered small-diameter vascular grafts. J Biomed Mater Res A 2009;90(1):205-216. 
3. Zhang WJ, Liu W, Cui L, Cao Y. Tissue engineering of blood vessel. $J$ Cell Mol Med. 2007;11(5):945-957.

4. Desai ND, Naylor CD, Kiss A, et al. Impact of patient and target-vessel characteristics on arterial and venous bypass graft patency: insight from a randomized trial. Circulation. 2007;115(6):684-691.

5. Zhou M, Liu Z, Wei Z, et al. Development and validation of small-diameter vascular tissue from a decellularized scaffold coated with heparin and vascular endothelial growth factor. Artif Organs. 2009;33(3):230-239.

6. Kong X, Han B, Wang H, Li H, Xu W, Liu W. Mechanical properties of biodegradable small-diameter chitosan artificial vascular prosthesis. J Biomed Mater Res A. 2012;100(8):1938-1945.

7. Lee SJ, Liu J, Oh SH, Soker S, Atala A, Yoo JJ. Development of a composite vascular scaffolding system that withstands physiological vascular conditions. Biomaterials. 2008;29(19):2891-2898.

8. Ghezzi CE, Marelli B, Muja N, Nazhat SN. Immediate production of a tubular dense collagen construct with bioinspired mechanical properties. Acta Biomater. 2012;8(5):1813-1825.

9. Li WJ, Laurencin CT, Caterson EJ, Tuan RS, Ko FK. Electrospun nanofibrous structure: a novel scaffold for tissue engineering. J Biomed Mater Res. 2002;60(4):613-621.

10. Bhattarai SR, Bhattarai N, Yi HK, Hwang PH, Cha DI, Kim HY. Novel biodegradable electrospun membrane: scaffold for tissue engineering. Biomaterials. 2004;25(13):2595-2602.

11. Chew SY, Wen J, Yim EK, Leong KW. Sustained release of proteins from electrospun biodegradable fibers. Biomacromolecules. 2005; 6(4):2017-2024.

12. Tillman BW, Yazdani SK, Lee SJ, Geary RL, Atala A, Yoo JJ. The in vivo stability of electrospun polycaprolactone-collagen scaffolds in vascular reconstruction. Biomaterials. 2009;30(4):583-588.

13. Han F, Jia X, Dai D, et al. Performance of a multilayered small-diameter vascular scaffold dual-loaded with VEGF and PDGF. Biomaterials. 2013;34(30):7302-7313.

14. Heydarkhan-Hagvall S, Schenke-Layland K, Dhanasopon AP, et al. Three-dimensional electrospun ECM-based hybrid scaffolds for cardiovascular tissue engineering. Biomaterials. 2008;29(19): 2907-2914.

15. Boland ED, Matthews JA, Pawlowski KJ, Simpson DG, Wnek GE, Bowlin GL. Electrospinning collagen and elastin: preliminary vascular tissue engineering. Front Biosci. 2004;9:1422-1432.

16. Matthews JA, Wnek GE, Simpson DG, Bowlin GL. Electrospinning of collagen nanofibers. Biomacromolecules. 2002;3(2):232-238.

17. McKenna KA, Hinds MT, Sarao RC, et al. Mechanical property characterization of electrospun recombinant human tropoelastin for vascular graft biomaterials. Acta Biomater. 2012;8(1):225-233.

18. Zhang X, Thomas V, Xu Y, Bellis SL, Vohra YK. An in vitro regenerated functional human endothelium on a nanofibrous electrospun scaffold. Biomaterials. 2010;31(15):4376-4381.

19. Yin A, Zhang K, McClure MJ, et al. Electrospinning collagen/chitosan/ poly(L-lactic acid-co-epsilon-caprolactone) to form a vascular graft: mechanical and biological characterization. J Biomed Mater Res A. 2013;101(5):1292-1301.

20. Sarkar S, Salacinski HJ, Hamilton G, Seifalian AM. The mechanical properties of infrainguinal vascular bypass grafts: their role in influencing patency. Eur J Vasc Endovasc Surg. 2006;31(6):627-636.
21. Feng B, Tu H, Yuan H, Peng H, Zhang Y. Acetic-acid-mediated miscibility toward electrospinning homogeneous composite nanofibers of GT/ PCL. Biomacromolecules. 2012;13(12):3917-3925.

22. Huang HM, Wu SF, Ren H. Tissue-engineered graft constructed by self-derived cells and heterogeneous acellular matrix. J Zhejiang Univ Sci B. 2006;7(5):351-356.

23. Yue W, Pi QM, Zhang WJ, et al. Platelet endothelial cell adhesion molecule-1, stage-specific embryonic antigen-1, and Flk-1 mark distinct populations of mouse embryonic stem cells during differentiation toward hematopoietic/endothelial cells. Stem Cells Dev. 2010;19(12):1937-1948.

24. Zhang K, Wang H, Huang C, Su Y, Mo X, Ikada Y. Fabrication of silk fibroin blended P(LLA-CL) nanofibrous scaffolds for tissue engineering. J Biomed Mater Res A. 2010;93(3):984-993.

25. Raghunath J, Rollo J, Sales KM, Butler PE, Seifalian AM. Biomaterials and scaffold design: key to tissue-engineering cartilage. Biotechnol Appl Biochem. 2007;46 Pt 2:73-84.

26. Dvir T, Timko BP, Kohane DS, Langer R. Nanotechnological strategies for engineering complex tissues. Nat Nanotechnol. 2011;6(1):13-22.

27. Duan H, Feng B, Guo X, et al. Engineering of epidermis skin grafts using electrospun nanofibrous gelatin/polycaprolactone membranes. Int J Nanomedicine. 2013;8:2077-2084.

28. Xue J, Feng B, Zheng R, et al. Engineering ear-shaped cartilage using electrospun fibrous membranes of gelatin/polycaprolactone. Biomaterials. 2013;34(11):2624-2631.

29. He X, Fu W, Feng B, et al. Electrospun collagen-poly(L-lactic acidco-epsilon-caprolactone) membranes for cartilage tissue engineering. Regen Med. 2013;8(4):425-436.

30. Xu Y, Wu J, Wang H, et al. Fabrication of electrospun poly(L-lactideco-epsilon-caprolactone)/collagen nanoyarn network as a novel, three-dimensional, macroporous, aligned scaffold for tendon tissue engineering. Tissue Eng Part C Methods. 2013;19(12):925-936.

31. Gilbert TW, Stewart-Akers AM, Badylak SF. A quantitative method for evaluating the degradation of biologic scaffold materials. Biomaterials. 2007;28(2):147-150.

32. Sung HJ, Meredith C, Johnson C, Galis ZS. The effect of scaffold degradation rate on three-dimensional cell growth and angiogenesis. Biomaterials. 2004;25(26):5735-5742.

33. Alsberg E, Kong HJ, Hirano Y, Smith MK, Albeiruti A, Mooney DJ. Regulating bone formation via controlled scaffold degradation. J Dent Res. 2003;82(11):903-908.

34. Gong YY, Xue JX, Zhang WJ, Zhou GD, Liu W, Cao Y. A sandwich model for engineering cartilage with acellular cartilage sheets and chondrocytes. Biomaterials. 2011;32(9):2265-2273.

35. Fernandez J, Etxeberria A, Sarasua JR. Effects of repeat unit sequence distribution and residual catalyst on thermal degradation of poly(Llactide/epsilon-caprolactone) statistical copolymers. Polym Degrad Stab. 2013;98(7):1293-1299.

36. Larranaga A, Sarasua J-R. Effect of bioactive glass particles on the thermal degradation behaviour of medical polyesters. Polym Degrad Stab. 2013;98(3):751-758.

37. Zheng R, Duan H, Xue J, et al. The influence of gelatin/PCL ratio and 3-D construct shape of electrospun membranes on cartilage regeneration. Biomaterials. 2014;35(1):152-164.
International Journal of Nanomedicine

\section{Publish your work in this journal}

The International Journal of Nanomedicine is an international, peerreviewed journal focusing on the application of nanotechnology in diagnostics, therapeutics, and drug delivery systems throughout the biomedical field. This journal is indexed on PubMed Central, MedLine, CAS, SciSearch ${ }^{\circledR}$, Current Contents ${ }^{\circledR} /$ Clinical Medicine,

\section{Dovepress}

Journal Citation Reports/Science Edition, EMBase, Scopus and the Elsevier Bibliographic databases. The manuscript management system is completely online and includes a very quick and fair peer-review system, which is all easy to use. Visit http://www.dovepress.com/ testimonials.php to read real quotes from published authors. 